\title{
Evaluation of the reliability of reinforced concrete beams on a stochastically inhomogeneous elastic foundation under the action of a non-stationary random load
}

\author{
Pavel Deminov ${ }^{*}$ and Andrey Danilkiv
}

Moscow State University of Civil Engineering, Yaroslavskoe shosse, 26, Moscow, 129337, Russia

\begin{abstract}
The results of the evaluation of the reliability of reinforced concrete beams lying on an elastic foundation are presented. The load on the beam is considered as a non-stationary random function, the elastic properties of the foundation are described as a stationary random function. Beam stiffness is considered as a random variable depending on the cubic strength of concrete. To solve the beam bending equation on an elastic foundation with random properties and a loaded non-stationary random load, the small parameter method and the method of spectral representations are used. The obtained probability characteristics of the probability density distribution of bending moments allow us to find the probability of failure of a reinforced concrete beam on an elastic stochastically inhomogeneous foundation.
\end{abstract}

By the reliability of the beam e, we mean the probability of nondestructive over a normal section of a reinforced concrete beam with random stiffness $B(R)$ lying on an elastic stochastic heterogeneous foundation with a Winkler ratio $C(x)$ and a random nonstationary load loaded $q(x)$ :

$$
e=1-e_{p}
$$

Destruction of a reinforced concrete beam over a normal section occurs if $M(x)>$ $M_{u l t}\left(R, \sigma_{\mathrm{T}}\right)$, where $M_{u l t}\left(R, \sigma_{\mathrm{T}}\right)$ is the random value of the bearing capacity of the beam, and $M(x)$ is the normally distributed bending moments in characteristic sections beams depending on random parameters $B(R), q(x), C(x)$. The probability of failure of a reinforced concrete beam in a normal section in this case will be equal to:

$$
e_{p}=\iiint_{-\infty}^{\infty} \int p_{\sigma_{\mathrm{T}}}\left(\sigma_{\mathrm{T}}\right) p_{R}(R) p_{q}(q) p_{C}(C)\left[\int_{M_{u l t}\left(R, \sigma_{\mathrm{T}}\right)}^{\infty} p_{M}(M, R, q, C) d M\right] d \sigma_{\mathrm{T}} d R d q d C,
$$

where $p_{R}(R), p_{\sigma_{\mathrm{T}}}\left(\sigma_{\mathrm{T}}\right), p_{q}(q), p_{C}(C)$ - probability density functions of cubic concrete strength $R$ and the yield strength of reinforcement $\sigma_{\mathrm{T}}$, random non-stationary load $q(x)$ and random function of coefficient of the foundation (bed ratio) $C(x)$;

$p_{M}(M, R, q, C)$ - probability density function of bending moments in the beam.

\footnotetext{
* Corresponding author: kittaobao@mail.ru
} 
All random parameters are accepted distributed according to the normal Gaussian distribution.

Issues related to the evaluation of the reliability of reinforced concrete structures were considered in [1-6].

In [7] the parameters of the distribution of deflections and bending moments in a reinforced concrete beam lying on an elastic foundation were constructed. In this case, the load on the beam was considered as a stationary random function, and the elastic properties of the foundation were described by the Winkler model, which was also considered as a stationary random function.

In the practice of designing foundation structures, the task of bending a reinforced concrete foundation beam under the action of a non-stationary load, which is a function of the coordinate, is quite often encountered.

The average values of the random Winkler coefficient of the foundation, the external load and deflections of the beam are denoted respectively by $\langle C\rangle,\langle q(x)\rangle,\langle w(x)\rangle$ and the correlation functions of these parameters are denoted as $K_{C}\left(x-x^{\prime}\right), K_{q}\left(x, x^{\prime}\right)$, $K_{w}\left(x, x^{\prime}\right)$. Suppose also that the cube strength of concrete $R$ has received a random implementation, which corresponds to the flexural rigidity of the beam $B_{0}$.

The equation of the bend of a bar loaded with an arbitrary load and lying on an elastic foundation with Winkler coefficient has the well-known form:

$$
B_{0} \frac{d^{4} w(x)}{d x^{4}}+C(x) \cdot w(x)=q(x)
$$

To solve equation (3), we will use the small parameter method in the form, which was done in [7].

We represent the function of the deflection of the beam $w(x)$, as well as the function of repelling the foundation $C(x)$ and the load $q(x)$ in the form:

$$
\begin{gathered}
q(x)=<q(x)>+\vartheta \cdot q_{1}(x) \\
C(x)=<C>+\vartheta \cdot C_{1}(x) \\
w(x)=<w(x)+\vartheta \cdot w_{1}(x)+\vartheta^{2} \cdot w_{2}(x)+\cdots=\sum_{n=0}^{N} \vartheta^{n} w_{n}(x)
\end{gathered}
$$

here $n=0,1,2, \ldots, N \rightarrow \infty(x)$;

$\vartheta$ - a small parameter that, after all calculations are performed, is equal to 1 .

Substituting expressions (4), (5) and (6) into equation (3) and equating the terms with the same degree of small parameter, we turn to the system of differential equations:

$$
\begin{aligned}
& B_{0}(R) \frac{d^{4}<w>}{d x^{4}}+<C>\cdot<w(x)>=<q(x)> \\
& B_{0}(R) \frac{d^{4} w_{1}(x)}{d x^{4}}+<C>\cdot w_{1}(x)=q_{1}(x)-<w(x)>\cdot C_{1}(x) \\
& \quad \ldots \ldots \ldots \ldots \ldots \ldots \\
& B_{0}(R) \frac{d^{4} w_{n}(x)}{d x^{4}}+<C>\cdot w_{n}(x)=-w_{n-1}(x) \cdot C_{1}(x) .
\end{aligned}
$$

We confine ourselves to solving the first two equations (7) and (8) from the above system of equations. In them, the functions $w_{1}(\mathrm{x}), q_{1}(\mathrm{x})$ и $C_{1}(x)$ are centered random functions. Obviously, solving the equation (7) of the above system of equations gives us the expectation of the beam deflections.

To solve equation (8) of the resulting system, we use the method of spectral representations. Given the well-known fact that the correlation function and the spectral density of a random process make up a pair of Fourier transforms, known as the WienerKhinchin theorem, we will have the following expression for the impedance of equation (8): 


$$
J(\omega)=B_{0} \omega^{4}+\langle C\rangle,
$$

then the transfer function $H(\omega)$ of equation (8) will be:

The right-hand side of equation $(8)$ can be written as:

$$
\Phi(x)=q_{1}(x)-<w(x)>\cdot C_{1}(x) .
$$

The generalized spectral density of a nonstationary random function $\Phi(x)$ is represented as follows:

$$
S_{\Phi}\left(\omega, \omega_{1}\right)=<w(x)>^{2} S_{C}(\omega)+S_{q}\left(\omega, \omega_{1}\right) .
$$

In expression (13), the first term is the spectral density of the base stiffness. The correlation function of the Winkler modulus can be taken in the form:

$$
\begin{gathered}
K_{c}\left(x-x^{\prime}\right)=D_{c} \exp \left[-v^{n}\left|x-x^{\prime}\right|^{n}\left\{\cos ^{k}\left[\varphi\left(x-x^{\prime}\right)\right]+\right.\right. \\
\left.+\left(\frac{v}{\varphi}\right)^{m} \sin ^{m}\left[\varphi\left|x-x^{\prime}\right|\right]\right\},
\end{gathered}
$$

where $D_{c}$ - variance of Winkler coefficient of foundation;

$v$ и $\varphi$ - coefficients, the method for determining which is given in [8];

$x-x^{\prime}$ - the distance between arbitrary correlated sections of the beam;

$k, m$ - coefficients that can be taken as equal to 0 or 1 ; random base.

$n$ - coefficient taken as equal to 1 or 2 , depending on the properties of the

In [12], according to the results of soil testing, it was recommended to take the coefficients $=2, \mathrm{k}=1, \mathrm{~m}=0$, while the experimental and approximate curves shown in Fig. 1, as showed a good match.

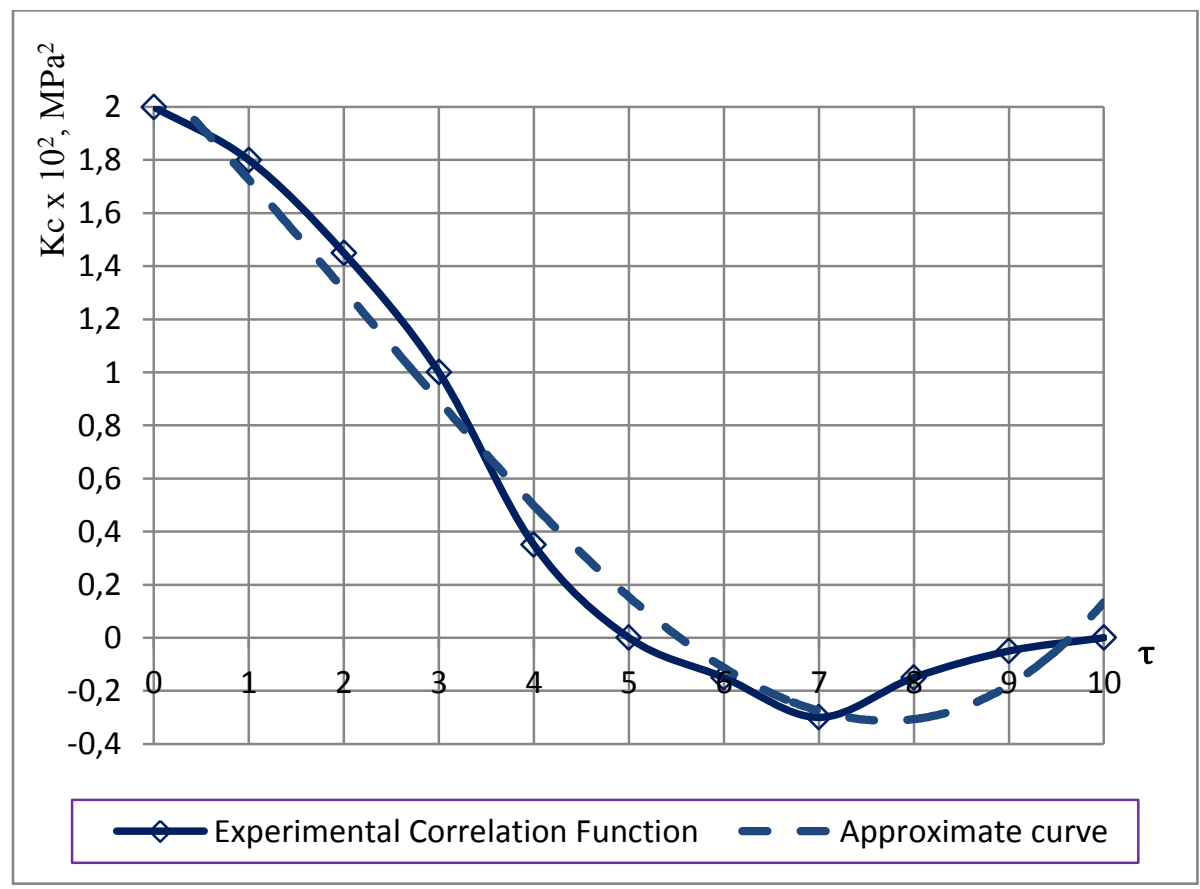

Fig.1. Experimental and approximating correlation functions

In this case, the spectral density of the Winkler modulus of the foundation will have the form of rational functions: 


$$
S_{C}=D_{c} \frac{v^{2}}{\pi}\left\{\frac{1}{\left[(\omega-\varphi)^{2}+v^{4}\right]}+\frac{1}{\left[(\omega+\varphi)^{2}+v^{4}\right]}\right\} .
$$

The second term in expression (13) is the spectral density of the load, which is determined by the generalized Wiener-Khinchin transform [9]:

$$
S_{q}\left(\omega, \omega_{1}\right)=\frac{1}{4 \pi^{2}} \iint_{-\infty}^{\infty} K_{q}\left(x, x^{\prime}\right) e^{-i\left(\omega x-\omega_{1} x^{\prime}\right)} d x d x^{\prime} .
$$

Applying the inverse generalized Wiener-Khinchin transform [10, 11] with the expression for the impedance (10) of equation (8), we obtain the correlation function of the beam deflections:

$$
\begin{aligned}
K_{w}\left(x, x^{\prime}\right)= & 2<w(x)>^{2} \int_{0}^{\infty} \frac{S_{C}(\omega) \cos \left[\left(x-x^{\prime}\right)\right] d \omega}{\left|B_{0} \omega^{4}+<C>\right|^{2}}+ \\
& +\iint_{-\infty}^{\infty} \frac{S_{q}\left(\omega, \omega_{1}\right) e^{-i\left(\omega x-\omega_{1} x^{\prime}\right)} d \omega d \omega_{1}}{\left(B_{0} \omega_{1}^{4}+<C>\right)\left(B_{0} \omega^{4}+<C>\right)} .
\end{aligned}
$$

The correlation function of bending moments as the second derivative of the deflection function will be equal to:

$$
\begin{gathered}
K_{M}\left(x, x^{\prime}\right)=2 B_{0}{ }^{2}<w(x)>^{2} \int_{0}^{\infty} \frac{\omega^{4} S_{C}(\omega) \cos \left[\left(x-x^{\prime}\right)\right] d \omega}{\left|B_{0} \omega^{4}+<C>\right|^{2}}+ \\
+B_{0}{ }^{2} \iint_{-\infty}^{\infty} \frac{\omega^{2} \omega_{1}^{2} S_{q}\left(\omega, \omega_{1}\right) e^{-i\left(\omega x-\omega_{1} x^{\prime}\right)} d \omega d \omega_{1}}{\left(B_{0} \omega_{1}^{4}+<C>\right)\left(B_{0} \omega^{4}+<C>\right)}
\end{gathered}
$$

For the variance of bending moments, we will have at $x=x^{\prime}$ :

$$
\begin{aligned}
D_{M}(x)= & K_{M}(x, x)=2 B_{0}{ }^{2}<w(x)>^{2} \int_{0}^{\infty} \frac{\omega^{4} S_{C}(\omega) d \omega}{\left|B_{0} \omega^{4}+<C>\right|^{2}}+ \\
& +B_{0}^{2} \iint_{-\infty}^{\infty} \frac{\omega^{2} \omega_{1}^{2} S_{q}\left(\omega, \omega_{1}\right) e^{-i x\left(\omega-\omega_{1}\right)} d \omega d \omega_{1}}{\left(B_{0} \omega_{1}^{4}+<C>\right)\left(B_{0} \omega^{4}+<C>\right)}
\end{aligned}
$$

As expected, the variance of the bending moments turned out to be a function of the $x$ coordinate, i.e. output random process is non-stationary.

Consider special cases of loading the beam. Let the load be specified in a deterministic form and is a function of the coordinate $\boldsymbol{x}$, i.e. when $D_{q}=0$, in this case the correlation function of the beam deflections will be:

$$
K_{w}\left(x, x-x^{\prime}\right)=2<w(x)>^{2} \int_{0}^{\infty} \frac{S_{C}(\omega) \cos \left[\left(x-x^{\prime}\right)\right] d \omega}{\left|B_{0} \omega^{4}+<C>\right|^{2}},
$$

and the variance of the deflections of the beam will be equal at $x=x^{\prime}$ : 


$$
D_{w}(x)=2<w(x)>^{2} \int_{0}^{\infty} \frac{S_{C}(\omega) d \omega}{\left|B_{0} \omega^{4}+<C>\right|^{2}} .
$$

Thus, despite the fact that the load is a non-random function and Winkler coefficient of the foundation is a stationary random function, the result is obtained as a non-stationary random function.

Consider another case where the load is a quasi-stationary random function, in other words, we assume that the load expectation is a non-random function of $\boldsymbol{x}$, and the centered random component of the load is a stationary random function, i.e.

$$
<q>=<q(x)>; \quad D_{q}=\text { const. }
$$

In this case, the correlation function of the beam deflections will take the form:

$$
\begin{aligned}
K_{w}\left(x, x-x^{\prime}\right)= & 2<w(x)>^{2} \int_{0}^{\infty} \frac{S_{C}(\omega) \cos \left[\left(x-x^{\prime}\right)\right] d \omega}{\left|B_{0} \omega^{4}+<C>\right|^{2}}+ \\
& +\int_{0}^{\infty} \frac{S_{q}(\omega) \cos \left[\left(x-x^{\prime}\right)\right] d \omega}{\left|B_{0} \omega^{4}+<C>\right|^{2}},
\end{aligned}
$$

And the variance of the deflections of the beam will take the form:

$$
D_{w}(x)=2<w(x)>^{2} \int_{0}^{\infty} \frac{S_{C}(\omega) d \omega}{\left|B_{0} \omega^{4}+<C>\right|^{2}}+2 \int_{0}^{\infty} \frac{S_{q}(\omega) d \omega}{\left|B_{0} \omega^{4}+<C>\right|^{2}} .
$$

Similarly, the expressions for the correlation function and the variance of bending moments in the beam will be written:

$$
\begin{gathered}
K_{M}\left(x, x-x^{\prime}\right)=2 B_{0}{ }^{2}<w(x)>^{2} \int_{0}^{\infty} \frac{\omega^{4} S_{C}(\omega) \cos \left[\left(x-x^{\prime}\right)\right] d \omega}{\left|B_{0} \omega^{4}+<C>\right|^{2}}+ \\
+B_{0}{ }^{2} \int_{0}^{\infty} \frac{\omega^{4} S_{q}(\omega) \cos \left[\left(x-x^{\prime}\right)\right] d \omega}{\left|B_{0} \omega^{4}+<C>\right|^{2}} \\
\cdot \int_{0}^{\infty} \frac{\omega^{4} S_{C}(\omega) d \omega}{\left|B_{0} \omega^{4}+<C>\right|^{2}}+2 B_{0}{ }^{2} \int_{0}^{\infty} \frac{\omega^{4} S_{q}(\omega) d \omega}{\left|B_{0} \omega^{4}+<C>\right|^{2}} .
\end{gathered}
$$

The results obtained are used for the case of loading an infinitely long reinforced concrete beam with a number of concentrated loads $P_{k}$ with steps $x_{k}$ and expected values $\langle P\rangle_{k}$. The expected value of the deflections of the beam, obtained from equation (7) can be written in a known form:

$$
<w(x)>=\frac{1}{8 \beta^{3} B_{0}}\left\{\sum_{k=0}^{\infty}<P>_{k} e^{-\beta\left|x-x_{k}\right|}\left[\cos \beta\left(x-x_{k}\right)+\sin \beta\left|x-x_{k}\right|\right]\right\},
$$

where $\beta=\sqrt[4]{<C>/ 4 B_{0}}$; 
$l$ - distance between concentrated loads.

The expected value of bending moments is obtained by differentiating twice (27) with respect $\boldsymbol{x}$ :

$$
<M(x)>=\frac{1}{4 \beta}\left\{\sum_{k=0}^{\infty}<P>_{k} e^{-\beta\left|x-x_{k}\right|}\left[\cos \beta\left(x-x_{k}\right)-\sin \beta\left|x-x_{k}\right|\right]\right\} .
$$

Now we can construct the probability density function of bending moments, given that it was taken by us normal distribution and, thus, is completely determined by the expected value and variance:

$$
p_{M}(M)=\frac{1}{\sqrt{2 \pi D_{M}(x)}} \exp \left\{-\frac{\left[M-<M(x)>^{2}\right]}{2 D_{M}(x)}\right\} .
$$

Substituting the expression for the probability distribution density of bending moments (29) into the expression for the probability of failure of a reinforced concrete beam over a normal cross section (2) and considering that all the above calculations were made for a concrete implementation of the concrete cube strength $R$, which is a random variable with a Gaussian distribution $p_{R}(R)$ with parameters: expectation $\langle R\rangle$ and variance $D_{R}$ and on which the beam stiffness and the probability characteristics of bending moments depend, find the probability of jelly destruction reinforced concrete beams of normal cross section.

\section{References}

1. A.G. Tamrazyan The assessment of reliability of punching reinforced concrete beamless slabs under the influence of a concentrated force at high temperatures. Procedia Engineering, 153, p.715-720 (2016)

2. A.G. Tamrazyan, M.A. Orlova, Residual capacity of reinforced concrete beams with cracks, Housing construction, 6, p.32-34, (2015)

3. A.G. Tamrazyan, Reduce the impact of dynamic strength of concrete under fire conditions on bearing capacity of reinforced concrete columns, Appl. Mech. and Mater, 475-476, p.1563-1566, (2014)

4. A.G. Tamrazyan, Calculation of structural elements for a given reliability and normal distribution of load and carrying capacity. Vestnik MGSU, 10, p.109-115, (2012)

5. A.G. Tamrazyan, Concrete and reinforced concrete: problems and prospects, Industrial and civil engineering, 7, p.51-54, (2014)

6. A.G. Tamrazyan, Dynamic stability of a compressed reinforced concrete element as a viscoelastic rod, Vestnik MGSU, 1-2, p. 193-196, (2011)

7. P.D. Deminov, Estimation of distribution parameters of bending moments in a reinforced concrete beam on an elastic stochastically inhomogeneous base under the action of a random distributed load, News of universities, Technology textile industry, 5, (2019)

8. V.L. Blagonadezhin, E.P. Kudryavtsev, Statistical study of deformations of sandy foundations and pipelines of underground waveguide communication lines, Reports of the scientific and technical conference on the results of research work for 1964-1965 of Moscow Power Engineering Institute, p.78-86, (1965)

9. A.Ya. Khinchin, Theory of correlation of stationary statistical processes, Successes of Mathematical Sciences, 5, p. 42-51, (1938)

10. D.I. Kazakevich, Fundamentals of the theory of random functions in problems of hydrometeorology, Gidrometeoizdat publishing, p. 100-105, (1989)

11. S.M. Rytov, Introduction to statistical radiophysics, Part 1, Nauka publishing, p. 443445, (1976)

12. S.N. Raskatov, Calculation of beam and plate pile grillages on an elastic stochastic foundation, Ph.D thesis, Moscow Civil Engineering Institute (MISI), (1976) 